\title{
SOME NEW NONLINEAR POWERED GRONWALL-BELLMAN TYPE RETARDED INTEGRAL INEQUALITIES AND THEIR APPLICATIONS
}

\author{
ZIZUN LI AND WU-SHENG WANG
}

Abstract. The purpose of the this paper is to establish some new retarded integral inequalities of Gronwall-Bellman type, which generalizes some known integral inequalities. The inequalities given here can be used in the analysis of the qualitative properties of certain classes of differential equations and integral equations. We apply our result to the boundedness of the solutions of integral equations.

Mathematics subject classification (2010): 26D10, 26D15, 26D20.

Keywords and phrases: Retarded integral inequality, power, explicit bounds, boundedness.

\section{REFERENCES}

[1] T. H. GRonWALL, Note on the derivatives with respect to a parameter of the solutions of a system of differential equations, Ann Math. 20(1919)292-296.

[2] R. Bellman, The stability of solutions of linear differential equations, Duke Math. J. 10 (1943) 643-647.

[3] B. G. PAChPATte, On some generalizations of Bellman's lemma, J. Math. Anal. Appl. 5 (1975) 141150 .

[4] B. G. Pachpatte, Inequalities for Differential and Integral Equations, Academic Press, London, 1998.

[5] H. EI-Owaidy, A. Ragab, A. Abdeldaim, On some new integral inequalities of GronwallBellman type, Appl. Math. Comput. 106 (1999) 289-303.

[6] O. Lipovan, A retarded Gronwall-like inequality and its applications, J. Math. Anal. Appl. 252 (2000) 389-401.

[7] R. P. AgARwal, S. F. DENG, W. N. ZHANG, Generalization of a retarded Gronwall-like inequality and its applications, Appl. Math. Comput. 165 (2005) 599-612.

[8] O. Lipovan, Integral inequalities for retarded Volterra equations, J. Math. Anal. Appl. 322 (2006) 349-358.

[9] R. P. Agarwal, Y. H. Kim, S. K. SEN, New retarded integral inequalities with applications, J. Inequal. Appl. 2008 (2008) Artical ID 908784.

[10] A. Abdeldaim, M. Yakout, On some new integral inequalities of Gronwall-Bellman-Pachpatte type, Appl. Math. Comput. 217 (2011) 7887-7899.

[11] J. ZHOU, J. SHEN, W. N. ZHANG, A powered Gronwall-type inequality and applications to stochastic differential equations, Discrt. Continu. Dyna. Syst. 36 (12) (2016) 7207-7234.

[12] A. ABDELDAim, A. A. EI-DEEB, On generalized of certain retarded nonlinear integral inequalities and its applications in retarded integro-differential equations, Appl. Math. Comput. 256 (2015) 375380 .

[13] A. AbDeldaim, Nonlinear retarded integral inequalities of Gronwall-Bellman type and applications, J. Math. Inequal. 10(1) (2016) 285-299.

[14] D. D. Bainov And P. Simeonov, Integral Inequalities and Applications, Kluwer Academic Publishers, Dordrecht, 1992.

[15] A. A. EI-DEEB, R. G. AhMeD, On some generalizations of certain nonlinear retarded integral inequalities for Volterra-Fredholm integral equations and their applications in delay differential equations, J. Egypt. Math. Soc. 25 (2017) 279-285. 
[16] R. A. C. Ferreira, D. F. M. Torres, Generalized retarded integral inequalities, Appl. Math. Let. 22 (2009) 876-881.

[17] F. C. JiAng AND F.W. MENG, Explicit bounds on some new nonlinear integral inequalities with delay, J. Comput. Appl. Math. 205 (2007) 479-486.

[18] M. Kuczma, An introduction to the theory of functional equations and inequalities: Cauchy's equation and Jensen's inequality, University of Katowice, Katowice, 1985.

[19] V. Lakshmikantham And S. Leela, Differential and Integral Inequalities, Theory and Applications, Academic Press, New York, 1969.

[20] W. N. Li, M. A. HAN, F. W. Meng, Some new delay integral inequalities and their applications, J. Comput. Appl. Math. 180 (2005) 191-200.

[21] Z. Z. LI, W. S. WANG, Explicit bounds of unknown function of some new weakly singular retarded integral inequalities for discontinuous functions and their applications, J. Inequal. Appl. 2017 (287) (2017) 20 pages.

[22] O. Lipovan, A retarded Gronwall-like inequality and its applications, J. Math. Anal. Appl. 252 (2000) 389-401.

[23] H. D. Liu, F. W. Meng, Some new generalized Volterra-Fredholm type discrete fractional sum inequalities and their applications, J. Inequal. Appl. 2016 (213) (2016) 16 pages.

[24] F. W. Meng, J. Shao, Some new Volterra-Fredholm type dynamic integral inequalities on time scales, Appl. Math. Comput. 223 (2013) 444-451.

[25] H. El-Owaidy, A. Ragab, W. Abuelela, A. A. El-Deeb, On some new nonlinear integral inequalities of Gronwall-Bellman type, Kyungpook Math. J. 54 (2014) 555-575.

[26] B. G. PachPatte, Explicit Bounds on certain integral inequalities, J. Math. Anal. Appl. 267 (2002) 48-61.

[27] H. SAno And N. Kunimatsu, Modified Gronwall's inequality and its application to stabilization problem for semilinear parabolic systems, Systems Control Lett. 22 (1994) 145-156.

[28] L. L. WAN, R. XU, Some generalized integral inequalities and there applications, J. Math. Inequal. 7 (3) (2013) 495-511.

[29] W. S. WANG, R. C. LUO, Z. Z. LI, A new nonlinear retarded inequality and its applications, J. Inequal. Appl. 2010 (8)(2010) Art. ID 462163.

[30] R. XU, X. T. MA, Some new retarded nonlinear Volterra-Fredholm type integral inequalities with maxima in two variables and their applications, J. Inequal. Appl. 2017 (187) (2017) 25 pages. 